\title{
An Augmented Reality Weather System
}

\author{
Marko Heinrich* \\ University of Koblenz-Landau
}

\author{
Bruce H. Thomas ${ }^{\dagger}$ \\ Wearable Computer Lab \\ University of South Australia
}

\author{
Stefan Mueller \\ University of Koblenz-Landau
}

\author{
Christian Sandor ${ }^{\S}$ \\ Wearable Computer Lab \\ University of South Australia
}

\begin{abstract}
This demo presents ARWeather, a simulation application, which can simulate three types of precipitation: rain, snow, and hail. ARWeather was developed and deployed on the Tinmith wearable computer system to enable autonomous and free movement of the user.
\end{abstract}

Keywords: Augmented Reality, Visual Weather Simulation

\section{INTRODUCTION}

Modern computer games often use weather simulations to add texture and realism to the gameplay, This demo ${ }^{1}$, which accompanies our poster[1], explores the use of outdoor Augmented Reality (AR) to provide the impression of simulated weather. Imagine it is a bright sunny day, and with the flick of a switch it is now pouring rain. A user equipped with a wearable computer can walk and move freely inside the artificial weather and enjoy a new experience. They get a 360 degree impression that they are in the middle of the occurring weather. We currently support the simulation of three types of precipitation: rain, snow, and hail (see Figure 1).

Our demo is running on the Tinmith mobile AR system. The system is based on a modified Toshiba Tecra M5 with a PentiumM $2.0 \mathrm{GHz}, 2$ GB RAM, NVIDIA GeForce 6600 graphics chipset, 802.11 wireless networking, and Bluetooth. The computer system is mounted on the back of the belt, with battery power for 2-3 hours. Headphones are fixed to the inside the helmet, so ambient sounds can still be heard. The Tinmith system employs video see-through technology. A 640x480 pixel 30fps Point Gray Firefly firewire video camera is attached on the front of the helmet. A GPS antenna and an InterSense InertiaCube 3 inertial orientation sensor are mounted on the helmet. The GPS receiver has sub-50 $\mathrm{cm}$ accuracy and is used to track the position of the users head outdoors.

Software highlights of this demo include: GPU-accelerated particle systems and video processing, spatial audio with OpenAL, and physics-based interaction of particles with the environment (e.g., hail bounces of the ground).

To date, there have been a very limited number of investigations into the visual simulation of weather for outdoor AR. A visual change of the real weather conditions may be used as a supplement to training simulations for search and rescue teams or military training. Another big application area is entertainment and art. The example screenshots (see Figure 1) underline that we can create a surreal user experience; i.e., seeing and hearing a snow storm in an area where it usually never snows.

\footnotetext{
*e-mail: marko.heinrich@uni-koblenz.de

†e-mail: bruce.thomas@unisa.edu.au

† e-mail: stefan.mueller@uni-koblenz.de

§e-mail: christian.sandor@unisa.edu.au
}

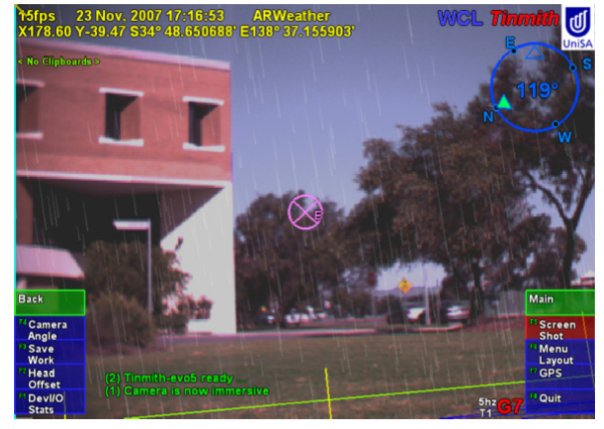

(a) Rain

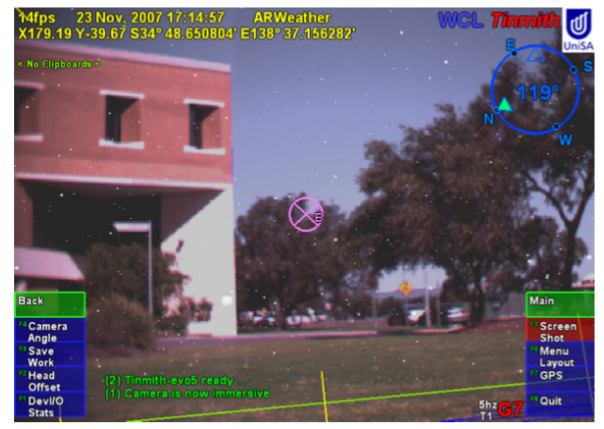

(b) Snow

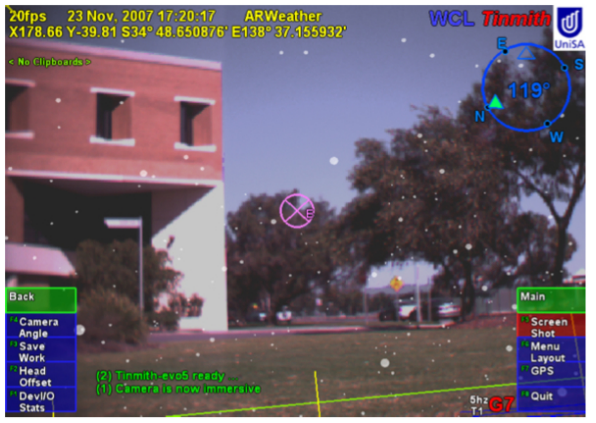

(c) Hail

Figure 1: The user's view through a head-worn display.

\section{REFERENCES}

[1] M. Heinrich, B. H. Thomas, and S. Mueller. ARWeather-An Augmented Reality Weather System. In Poster at IEEE and ACM International Symposium on Mixed and Augmented Reality, Cambridge, England, September 2008.

\footnotetext{
${ }^{1} \mathrm{~A}$ video for this demo is available at: http://wcl.ml.unisa. edu.au/ sandorc/ismar08/ismar08-arweather.avi
} 